\title{
High-Altitude Particle Acceleration and Radiation in Pulsar Slot Gaps
}

\author{
Alex G. Muslimov ${ }^{1} \&$ Alice K. Harding ${ }^{2}$
}

\begin{abstract}
We explore the pulsar slot gap (SG) electrodynamics up to very high altitudes, where for most relatively rapidly rotating pulsars both the standard small-angle approximation and the assumption that the magnetic field lines are ideal stream lines break down. We address the importance of the electrodynamic conditions at the SG boundaries and the occurrence of a steady-state drift of charged particles across the SG field lines at very high altitudes. These boundary conditions and the cross-field particle motion determine the asymptotic behavior of the scalar potential at all radii from the polar cap (PC) to near the light cylinder. As a result, we demonstrate that the steady-state accelerating electric field, $E_{\|}$, must approach a small and constant value at high altitude above the $\mathrm{PC}$. This $E_{\|}$is capable of maintaining electrons moving with high Lorentz factors $(\sim$ a few $\times$ $10^{7}$ ) and emitting curvature $\gamma$-ray photons up to nearly the light cylinder. By numerical simulations, we show that primary electrons accelerating from the PC surface to high altitude in the SG along the outer edge of the open field region will form caustic emission patterns on the trailing dipole field lines. Acceleration and emission in such an extended SG may form the physical basis of a model that can successfully reproduce some pulsar high-energy light curves.
\end{abstract}

Subject headings: acceleration of particles - gamma rays: theory - pulsars: general - radiation mechanisms: nonthermal — stars: neutron

\section{INTRODUCTION}

There is no doubt that pulsars are accelerating particles up to relativistic energies in their magnetospheres, and that these particles are primarily responsible for the pulsar radio- to high-energy non-thermal emission. It is also believed that the energetics of this acceleration,

\footnotetext{
${ }^{1}$ ManTech International Corporation, Lexington Park, MD 20653

${ }^{2}$ Laboratory of High Energy Astrophysics, NASA/Goddard Space Flight Center, Greenbelt, MD 20771
} 
as well as the main physical processes involved in production of high-energy photons, are more or less understood. However, the ambiguity in interpretation of pulsar timing observations in terms of emission site mapping in a pulsar magnetosphere makes it difficult to answer the basic question of where the pulsar high-energy emission originates. In their recent attempt to explain the observed high-energy light curves of pulsars, Dyks \& Rudak (2003) concentrated on a purely geometrical model by postulating that the emission is produced in a relatively narrow region along the last open magnetic field lines of a pulsar magnetosphere. The interesting result of their study is the occurrence of caustic emission zones (Morini 1983), i.e. the phase shifts of radiation emitted at radii between $\sim 0.1-0.7$ times the light cylinder radius, parallel to field lines on the trailing edge of the polar cap ( $\mathrm{PC})$, are cancelled by phase shifts due to relativistic effects of aberration and time-of-flight. Radiation emitted over a large range of altitudes thus arrives in phase, forming two narrow peaks in the light curves, very similar to those of known $\gamma$-ray pulsars (e.g. Thompson 2001).

In our previous paper (Muslimov \& Harding 2003 [MH03]) we began discussing the regime of acceleration of particles and production of high-energy emission within the pulsar slot gap (SG), a narrow region on the boundary of the open field lines, where the electric field drops to zero. The SG is a pair-free region of slower acceleration, in which the parallel electric field is unscreened. Pair cascades develop along the inner edge of the SG at several stellar radii above the NS surface. Even though the SG regime in pulsars was originally introduced in the electrodynamic model of Arons \& Scharlemann (1979), it was not considered a viable high-energy emission region (see e.g. Arons 1996). The revised version of the SG regime proposed by $\mathrm{MH} 03$ incorporates the effect of relativistic frame dragging (Muslimov \& Tsygan 1992 [MT92]) and, more importantly, the effect of SG boundaries on the strength of the accelerating electric field within the SG. MH03 demonstrated that the primary electrons tend to accelerate up to higher altitudes before pair production begins, and pair cascades continue along the inner boundary of the SG until the magnetic field becomes too low. The resulting radiation from the pair cascades forms a wide, hollow cone of high-energy radiation due to the flaring of field lines. Adhering to the small-angle approximation, MH03 restricted their study of the SG regime to altitudes less than four-five stellar radii. However, since the parallel electric field in the SG is not screened on field lines close to the open-field boundary, acceleration may continue to much higher altitudes. Particle acceleration and radiation in such an extended SG may therefore provide a physical basis for the two-pole caustic model of Dyks \& Rudak (2003).

Formation of a SG requires the production of enough pair multiplicity to screen the parallel electric field above the pair formation front. We have found from our previous studies (Harding \& Muslimov 2001 [HM01], 2002 [HM02]) that the youngest and most energetic pulsars can produce pairs from curvature radiation (CR) of primary electrons, which are 
numerous enough to screen the electric field. Older, less energetic pulsars, those below the CR pair death line, can produce only pairs from inverse Compton radiation of primary electrons scattering thermal X-rays from the NS surface. The inverse Compton pairs are not numerous enough to completely screen the parallel electric field. A necessary condition for formation of a $S G$ is thus the ability to produce pairs from $C R$, and the expression for the CR death line (given by Eqn [52] of HM02) defines the boundary in the P- $\dot{P}$ diagram of pulsars capable of having SGs. Such pulsars include the Crab, Vela, Geminga and most of the $\gamma$-ray pulsars detected by EGRET, but not the majority of millisecond pulsars.

The extension of the regime of SG acceleration to much higher altitudes is the main subject of the present paper. In the Sections below we outline our approach to constructing an appropriate steady-state physical solution that can be used up to very high altitudes in the SG. We also discuss the immediate consequences of our proposed extended SG solution: acceleration of particles (electrons, positrons) and high-energy emission up to nearly lightcylinder radius, and the possibility of occurrence of high-altitude caustic emission on trailing field lines.

The paper is organized as follows. In $\S 2$ we discuss the electrodynamics within the SG regions of pulsars. We address the physical constraints on the scalar potential (\$2.1) and equipotentiality of SG boundaries and derivation of effective Poisson's equation ( $\$ 2.2)$ in the outermost section of SG. In $\S 3$ we present the electrodynamic solution within the SG at very high altitudes. In $\S 3.1$ we illustrate the possibility of extended acceleration within SG in the regime where the acceleration is balanced by the curvature-radiation reaction. Our numerical calculations are discussed in $\$ 3.2$. Finally, in $\S 4$ we discuss our main results and draw our principal conclusions.

\section{Steady State SG Electrodynamics}

In the frame of reference rigidly corotating with a neutron star (NS), where the magnetic field is stationary (and having dipolar geometry), the general relativistic Maxwell's equations yield (see MT92)

$$
\mathbf{E}-\frac{1}{\alpha c}(\mathbf{w}-\mathbf{u}) \times \mathbf{B}=-\frac{1}{\alpha} \nabla \Phi,
$$

where $\mathbf{E}$ and $\mathbf{B}$ are the electric and magnetic fields defined in Zero-Angular-MomentumObserver (ZAMO) frame of reference (see Macdonald \& Thorne 1982), $\mathbf{u}$ is the rotational velocity, and $\mathrm{w}$ is the differential velocity of rotation of inertial frame of reference, $\Phi$ is the scalar potential, and the so-called geneneral-relativistic 'lapse function', $\alpha$, is defined below, right after expression (18). Taking the divergence of eq. (1) and making use of Maxwell 
equation

$$
\nabla \cdot \mathbf{E}=4 \pi \rho,
$$

we get the Poisson's equation for the scalar potential $\Phi$

$$
\nabla \cdot\left(\frac{1}{\alpha} \nabla \Phi\right)=4 \pi\left(\rho-\rho_{\mathrm{GJ}}\right),
$$

where

$$
\rho_{\mathrm{GJ}}=-\frac{1}{4 \pi c} \nabla \cdot\left[\frac{1}{\alpha}(\mathbf{u}-\mathbf{w}) \times \mathrm{B}\right]
$$

is the general relativistic expression for the Goldreich-Julian (GJ) charge density (cf. Goldreich \& Julian, 1969), and $\rho$ is the actual charge density of electrons determined by their relativistic flow along the magnetic field lines and which is fixed by the condition $E_{\|}=0$ at the stellar surface (see MT92 for details). Thus our electrodynamic description of charges streaming along the open field lines will imply the space-charge-limited flow approximation (at least near the stellar surface, within the radial distance of less than a few stellar radii).

Note that the 1.h.s. of eq. (1) can be treated as the effective electric field in the frame of reference rigidly corotating with the NS,

$$
\mathbf{E}^{\prime}=\mathbf{E}-\frac{1}{\alpha c}(\mathbf{w}-\mathbf{u}) \times \mathbf{B}
$$

The condition of absence of any electric field in the regions of the magnetosphere with closed field lines rigidly corotating with the NS, $\mathbf{E}^{\prime}=0$, necessarily implies that these regions should be filled with charges of density $\rho=\rho_{\mathrm{GJ}}$ (as a trivial solution of eq. [3]).

Our previously derived solutions (see MT92, Muslimov \& Harding 1997 [MH97]) for the case $\rho \lesssim \rho_{G J}$ were limited by a small-angle approximation and therefore cannot be justifiably used beyond the radial distances of about $\sim 3-4$ stellar radii above the PC surface (for a typical pulsar spin period). Recently, MH03 discussed the SG solution which is also formally limited to a small-angle approximation. This means that, because of the curving of the SG toward the magnetic equator, the solution derived in MH03 cannot be used for high altitudes, typically exceeding a few (or several, at most) stellar radii above the surface. Here we discuss the regime of steady-state acceleration within the SG extending up to very high altitudes, nearly approaching the light-cylinder. By addressing the basic physical conditions that are required for the occurrence of this regime, we observe that the standard concept of GJ charge density becomes inapplicable in the outermost section of the SG, where the effective GJ charge density gets significantly constrained by the requirement of equipotentiality of SG boundaries and by the effect of cross-field motion of charges.

We shall explore the SG solution in the outermost part of NS magnetosphere (but still within the light-cylinder) satisfying the same boundary conditions as those used in all our 
previous studies: equipotentiality of the SG surfaces, and zero-electric field condition at the PC surface. We propose the following ansatz for constructing the general solution extending from the innermost section up through the outermost section of the SG. By considering the fundamental property (see Section 2.1 below) of the scalar potential $\Phi$ at large (up to the light-cylinder radius) distances found by Mestel et al. (1985) together with the abovementioned boundary conditions (equipotentiality of SG surfaces and zero-electric field condition at the $\mathrm{PC}$ surface), we can unambiguosly constrain and determine the outermost solution for the potential $\Phi$. Then, by matching the outermost solution with the known innermost solution near the NS surface (presented in MH03) we can construct the approximate general solution applicable to both the innermost and outermost sections of the SG.

Note that any physically meaningful electrodynamic solution in the outermost part of the magnetosphere should take into account the effect of particle drift across the field lines or deviating of particle trajectories from magnetic field lines. This effect should unavoidably constrain the scalar potential $\Phi$, simply because in this region the field lines cannot be treated as characteristics or as stream lines for the flux of relativistically moving electrons. In a steady-state situation, it is reasonable to expect that the scalar potential $\Phi$ is a monotonically increasing (or decreasing, as in the case of acceleration of positive charges) and then saturating function of radial distance so that the outermost solution gradually matches the innermost one. In this study we demonstrate that in a steady-state situation, the constraint on $\Phi$ in the outermost region of the NS magnetosphere (but well within the light cylinder) along with the condition of equipotentiality of the SG surface allows us to derive an appropriate electrostatic solution. This solution implies initial (in the innermost section of a SG) boosting of electron acceleration over characteristic lenghtscale of $\sim 1-2$ stellar radii and subsequent extremely slow post-boost acceleration over lengthscale $\sim$ light-cylinder radius.

\subsection{Constraint on Potential $\Phi$ in the Outermost Section of SG}

The equation of motion of an electron of mass $m$ and charge $-e$ can be written as (see eq. [2.24] in Mestel et al. 1985)

$$
-\frac{e}{m}\left(\mathbf{E}+\frac{\mathbf{v}}{c} \times \mathbf{B}\right)=\mathbf{v} \cdot \nabla(\gamma \mathbf{v})=\nabla\left(\gamma c^{2}\right)-\mathbf{v} \times(\nabla \times(\gamma \mathbf{v}))
$$

where $\gamma=\left(1-v^{2} / c^{2}\right)^{-1 / 2}, \mathbf{v}$ is the electron velocity. In Mestel et al.'s notations,

$$
\mathbf{E}=-\nabla \phi
$$

and

$$
\frac{\mathbf{v}}{c} \times\left(\mathbf{B}-\frac{m c}{e} \nabla \times(\gamma \mathbf{v})\right)=\nabla \phi^{*}
$$


so that equation of motion, (6), translates into (see eq. [2.26] in Mestel et al. 1985)

$$
-e \phi+\gamma m c^{2}=-e \phi^{*}(S),
$$

where $S$ is the stream function.

Note that the scalar potential $\Phi$ defined by eq. (1) is a general-relativistic counterpart of the so-called 'non-corotational' potential $\psi$ (see e.g. eq. [2.5] in Mestel et al. 1985) introduced by Endean (1974), Mestel (1973), and Westfold (1981) and which can be defined via equation

$$
\nabla \psi=\nabla \phi-\frac{\boldsymbol{\Omega} \times \mathbf{r}}{c} \times \mathbf{B} .
$$

For relatively low altitudes $\Phi \approx \gamma\left(m c^{2} / e\right)$. For high altitudes (but still within the lightcylinder), as was first demonstrated by Mestel et al. (1985), the change in the angular momentum $\gamma m \Omega \tilde{r}^{2}$ (where $\tilde{r}$ is the radial cylindrical polar coordinate) of a streaming particle occurs only through the toroidal component of the magnetic force, requiring departure from strict flow along the field lines. The combination of energy and angular momentum integrals gives (in cylindrical polar coordinates in the axisymmetric case)

$$
\psi=\frac{m c^{2}}{e} \gamma\left(1-\frac{\Omega^{2} \tilde{r}^{2}}{c^{2}}\right)-\Gamma(S),
$$

where $\Gamma(S)$ is some function which is constant on stream lines and which is set at the stellar surface. Here the term $\propto \Omega^{2}$ is the so-called 'centrifugal-slingshot' term (see Mestel et al. 1985) arising from the flow of electrons across the field lines.

It is important to point out that within the domain of the SG the actual deviation from strict flow along (poloidal) $\mathbf{B}$ is of order

$$
\delta \approx \gamma m c^{2} /\left(e B \Delta l_{\mathrm{SG}}\right),
$$

where $\Delta l_{\mathrm{SG}} \sim \theta_{0} R \Delta \xi_{\mathrm{SG}} \sqrt{\eta_{*}}$ is the characteristic latitudinal SG thickness at dimensionless radial distance $\eta_{*}(\eta=r / R) ; \theta_{0}$ is the PC half-angle, $\theta_{0}=[\Omega R / f(1) c]^{1 / 2}$; and $\Delta \xi_{\mathrm{sG}}$ is the latitudinal SG thickness in units of $\xi\left(\xi=\theta / \theta_{0}\right.$ is the dimensionless colatitude of a PC field; see Section 3 and also MH03 for details). Thus, the characteristic dimensionless radial distance at which the magnitude of the deviation from strict flow along (poloidal) $\mathrm{B}$ reaches $\delta$, can be estimated as

$$
\eta_{*} \sim 0.6 \eta_{l c}\left(\Delta \xi_{\mathrm{sG}} \delta\right)^{2 / 5} \frac{R_{6}}{P_{0.1}}\left(\frac{R_{6}^{3} B_{12}^{2}}{\gamma_{7}^{2} P_{0.1}}\right)^{1 / 5},
$$

where $\eta_{l c}=c / \Omega R$ is the dimensionless radius of the light-cylinder; $\Omega=2 \pi / P$ is the angular velocity of NS rotation; $R$ and $P$ are the NS radius and spin period, respectively; $B_{12}=$ 
$B_{0} / 10^{12} \mathrm{G}, B_{0}$ is the surface value of NS magnetic field strength; $R_{6}=R / 10^{6} \mathrm{~cm}, P_{0.1}=$ $P / 0.1 \mathrm{~s}$, and $\gamma_{7}=\gamma / 10^{7}$.

For the parameters of the Crab pulsar (here we adopt the values: $B_{12}=8, R_{6}=1.6$, and $P_{0.1}=0.33$ ) and assuming $\delta \sim 0.05-0.1$, from formula (13) we get

$$
\eta_{*}=(0.3-0.4) \eta_{l c}\left(\frac{\Delta \xi_{\mathrm{SG}}}{0.01} \frac{3}{\gamma_{7}}\right)^{2 / 5}
$$

so that for the estimated value of $\Delta \xi_{\mathrm{sG}} \sim 0.05$ (see MH03) and for $\gamma_{7} \sim 3$ condition (13) is satisfied at $\eta_{*} \sim(0.5-0.7) \eta_{l c}$.

Formula (14) means that within the SG the effect of transfield motion becomes important already at $\eta \lesssim \eta_{l c}$ (for the Crab-like pulsars) and should be taken into account. This is significantly different from Mestel et al.'s model where a similar situation would occur well beyond the light cylinder (and where $\Delta l_{\mathrm{SG}}$ in formula [12] should be replaced by the light cylinder radius, $R_{l c}=c / \Omega$ ), because the lengthscale was the entire open field region rather than the narrow SG.

The fundamental consequence of eq. (11) is that in the region where the centrifugalslingshot effect becomes important (e.g. at $\eta \lesssim \eta_{l c}$ for the Crab-like pulsars) the electrons crossing the field lines begin picking up energy from the corotational part of the potential (from potential $\phi$, in Mestel et al.'s notation), so that in the regime of mostly transverse flow the change in potential (change in $\Gamma(S)$ ) across the field lines caused by rotation tends to balance the change in the corotational part of the potential. Suppose that the electrons are flowing with relativistic velocities along the magnetic field lines and entering the region where they are getting 'decoupled' from the magnetic field lines. Apparently, the solution of MH03 for $\rho$ and therefore for $\Phi$ will not be warranted in this region and especially in the region with predominantly transfield flow. However, we may justifiably assume that at the onset of the transfield flow regime, where the relativistic flow of electrons is still mostly along the magnetic field lines, the condition (see e.g. Mestel 1995, 1999)

$$
\mathbf{E}_{\perp}^{\prime} \approx \mathbf{E}_{\perp}+\frac{\boldsymbol{\Omega} \times \mathbf{r}}{c} \times \mathbf{B} \approx 0,
$$

'turns on', with $E_{\|} \ll E_{\perp}$ (for most acceleration scenarios we discuss in this paper $E_{\|}$is balanced by the $\mathrm{CR}$ reaction force). Further out in the magnetosphere the condition (15) may transform into the perfect MHD condition (see also Contopoulos, Kazanas \& Fendt, 1999), $\mathbf{E}+(\mathbf{v} / c) \times \mathbf{B}=0$, which is a good approximation as long as $E_{\|} \ll E_{\perp}$.

We suggest that the condition (15) together with the equipotentiality of the SG surfaces may in fact determine the behavior of potential $\Phi$ through the outermost section of the SG. 
It is important that at large radial distances $\mathbf{E}_{\perp}^{\prime}$ as given by eq. (5) is dominated by the term $(\Omega \times \mathbf{r} / c) \times \mathrm{B}$, which tends to produce significant electric potential drop across the SG, and as a result, would induce enormous surface charge on the SG boundaries. Also, it must be pointed out, that this term would tend to induce a strong component of $E_{\|}$with the polarity that may change with altitude and become opposite to that of the main component of $E_{\|}$, produced by a small imbalance between the GJ and actual charge densities in the innermost region, at altitudes within $\sim 1-2$ stellar radii. The occurrence of a strong component of $E_{\|}$with reversed polarity would unavoidably disrupt the continuous flow of electrons along the field lines and result in an essentially non-stationary regime of particle flow. However, in the steady-state situation we consider in this paper, the occurrence of cross-field motion of electrons would effectively screen out the excessive GJ space charge and short out the SG boundaries, thus maintaining them as equipotential. The latter means that condition (15) would be roughly satisfied in and beyond this region. Before we discuss how condition (15) can be explicitly incorporated into our electrostatic solution, let us discuss the consistency of this condition with the assumption of equipotentiality of SG boundaries all the way from the PC surface up to the very high altitudes (say, up to $\sim 0.1-0.5$ of the light-cylinder radius).

\subsection{Equipotentiality of SG Boundaries and Effective Poisson's Equation in the Outermost Section of SG}

Let us consider the cross-sectional area of a magnetic flux tube (of dipole field) emanating from the $\mathrm{PC}$ at radial distance $\eta(=r / R)$

$$
\mathcal{S}(\eta)=\mathcal{S}(1) \frac{f(1)}{f(\eta)} \eta^{3}
$$

where $f(\eta)$ is the general-relativistic correction factor, defined as (see e.g. MT92)

$$
f(\eta)=-3\left(\frac{\eta}{\varepsilon}\right)^{3}\left[\ln \left(1-\frac{\varepsilon}{\eta}\right)+\frac{\varepsilon}{\eta}\left(1+\frac{\varepsilon}{2 \eta}\right)\right],
$$

where $\eta=r / R$ is the dimensionless radial coordinate, $\varepsilon=r_{g} / R$, and $r_{g}$ is the gravitational radius of the NS. One can use approximate formula $f(\eta) \approx 1+0.75 x+0.6 x^{2}$ (where $x=\varepsilon / \eta$ ). For a canonical NS of 1.4 solar mass and $10 \mathrm{~km}$ radius $(\varepsilon=0.4) f(1) \approx 1.4$.

In this paper, as in our previous studies, we use the magnetic spherical polar coordinates $\left(\eta, \theta, \phi_{p c}\right)$. We also denote by $\chi$ the pulsar obliquity (angle between the NS rotation axis and magnetic moment). We will refer a 'normal polarity' pulsar as one having $0^{\circ} \leq \chi<90^{\circ}$ (north magnetic pole near north astrographic pole: $\Omega \cdot \mathbf{m}>0$, where $\mathbf{m}$ is the NS magnetic 
dipole moment), and a 'reversed polarity' pulsar as one having $90^{\circ}<\chi \leq 180^{\circ}$ (north magnetic pole near south astrographic pole: $\boldsymbol{\Omega} \cdot \mathbf{m}<0$ ).

We shall now introduce the flux of charges streaming with relativistic velocity through $\mathcal{S}(\eta)$,

$$
F=\alpha(\eta) c \mathcal{S}(\eta) \rho(\eta)
$$

where $\rho$ is the local charge density, and $\alpha=(1-\varepsilon / \eta)^{1 / 2}$ is the lapse function.

In a steady-state regime, well within the light cylinder, the flux $F$ should be constant along the individual magnetic flux tube as a consequence of charge continuity equation which implies that $\alpha \rho \propto B \propto \eta^{-3}$. In this case $F$ is a function of $\xi$ only (and not $\eta$ ). [The variable $\xi$ is equivalent to the Stokes stream function $S$ (see eq. [9]) used by Mestel et al.] However, at very high altitudes, for any stream line within the SG we can write that

$$
B=\frac{B_{0}}{f(1)} \frac{\beta}{\eta^{3}}
$$

where $\beta=\sqrt{1-3 \eta / 4 \eta_{l c}}$ is a factor that takes into account the change in the geometry of the flux tube as we move from the magnetic pole to the equator.

The explicit expressions (see e.g. MH97, for the derivation of these expressions) for the GJ charge density, $\rho_{\mathrm{GJ}}$, and actual charge density, $\rho$, may be written as

$$
\rho_{\mathrm{GJ}}=-\rho_{0} \frac{f(\eta)}{f(1)} \frac{1}{\alpha \eta^{3}}\left\{\left[a_{0}(\xi)+a_{1}(\eta, \xi)\right] \cos \chi+\left[b_{0}(\xi)+b_{1}(\eta, \xi)\right] \sin \chi \cos \phi_{\mathrm{pc}}\right\}
$$

and

$$
\rho \approx-\rho_{0} \frac{f(\eta)}{f(1)} \frac{1}{\alpha \eta^{3}} \beta(\eta)\left\{\left[a_{0}(\xi)+a_{1}(1, \xi)\right] \cos \chi+\left[b_{0}(\xi)+b_{1}(1, \xi)\right] \sin \chi \cos \phi_{\mathrm{pc}}\right\},
$$

respectively (see also MT92 for the exact expression for $\rho$ valid for arbitrary small altitudes and which is consistent with the radial profiles of potential $\Phi$ and $E_{\|}$). Here $\rho_{0}=\Omega B_{0} / 2 \pi c$, and

$$
\begin{gathered}
a_{0}=1, \quad a_{1}=-\frac{\kappa}{\eta^{3}}-\frac{3}{2} H(\eta) \sin ^{2} \theta \\
b_{0}=0, \quad b_{1}=\frac{3}{2} H(\eta) \sin \theta \cos \theta \\
H(\eta)=\frac{\varepsilon}{\eta}-\frac{\kappa}{\eta^{3}}+\frac{1}{(1-\varepsilon / \eta) f(\eta)}\left(1-\frac{3}{2} \frac{\varepsilon}{\eta}+\frac{\kappa}{2 \eta^{3}}\right) .
\end{gathered}
$$

Here $\kappa$ is the general-relativistic parameter characterizing the magnitude of the framedragging effect near the stellar surface measured in stellar rotation velocity, $\Omega$, and $\beta$ is 
defined right after eq. (19). These expressions are formally derived for arbitrarily large distances but still within the light cylinder. Well within the light cylinder, at $\eta \ll \eta_{l c}$ $\left(\eta_{l c} \sim \theta_{0}^{-2}\right)$, and in a small-angle approximation,

$$
a_{1} \approx-\frac{\kappa}{\eta^{3}}, \quad b_{1} \approx \frac{3}{2} \theta_{0} H(\eta) \sqrt{\eta \frac{f(1)}{f(\eta)}},
$$

which correspond to the expressions for $\rho$ and $\rho_{\mathrm{GJ}}$ we used in our previous papers for the situations where a small-angle approximation was in fact more than satisfactory. One can also use the approximate expression, $H(\eta) \approx 1-0.25 x-0.16 x^{2}-0.5\left(\kappa / \varepsilon^{3}\right) x^{3}\left(1-0.25 x-0.21 x^{2}\right)$, where $x=\varepsilon / \eta$. For a canonical NS [see also eq. $(17)$ ] $H(1) \approx 0.8$.

Now, the flux of effective GJ charges can be written as a sum of constant and varying with altitude components,

$$
F_{\mathrm{GJ}}=F_{\mathrm{GJ}, 0}+F_{\mathrm{GJ}, 1}
$$

where

$$
F_{\mathrm{GJ}, \mathrm{i}}=F_{0}\left(a_{i} \cos \chi+b_{i} \sin \chi \cos \phi_{\mathrm{pc}}\right), \quad i=0,1,
$$

and $F_{0}=-\rho_{0} c \mathcal{S}(1)$ is a constant factor depending on pulsar bulk parameters. Note that, according to the GJ reasoning (Goldreich \& Julian, 1969), the corotating region of the pulsar magnetosphere with closed magnetic field lines should be filled with charges of local density $\rho_{G J}$. This condition guarantees that any electric field which could be possibly generated in this region should be completely screened out. We do not intend to speculate on the dynamics of formation of the non-vacuum pulsar magnetosphere (see e.g. Krause-Polstorff \& Michel, 1985; Arons \& Spitkovsky, 2002), but we assume that the filling in of the closed field lines of the initially charge-starved magnetosphere with charges is most likely to occur along the field lines. Even more, since $F_{\mathrm{GJ}, 1}$ is a function of $\eta$, one may expect that this process develops in a non-stationary manner.

Let us now consider the flux $\Delta F$, corresponding to the charge imbalance between the GJ and actual local space-charge density, $\Delta \rho=\rho_{\mathrm{GJ}}-\rho$,

$$
\Delta F=F_{\mathrm{GJ}}-F \approx \alpha c \mathcal{S}(\eta)\left(\rho_{\mathrm{GJ}}-\rho\right) \approx \Delta F_{*}(\eta, \xi)-\beta(\eta) \Delta F_{*}(1, \xi),
$$

where

$$
\Delta F_{*}(\eta, \xi)=F_{0}\left[a_{1}(\eta, \xi) \cos \chi+b_{1}(\eta, \xi) \sin \chi \cos \phi_{\mathrm{pc}}\right]
$$

Using the explicit expressions for $a_{1}$ and $b_{1}$ (see eqs [22], [23]) we can write

$$
\begin{aligned}
\Delta F \approx & F_{0}\left\{\left[\kappa\left(\beta-\frac{1}{\eta^{3}}\right)+1-\beta+\frac{3}{2} H(1) \theta_{0}^{2}\left(\beta-\eta \frac{f(1)}{f(\eta)} \frac{H(\eta)}{H(1)}\right)\right] \cos \chi+\right. \\
& \left.\frac{3}{2} H(1) \theta_{0}\left(\frac{H(\eta)}{H(1)} \sqrt{\eta \frac{f(1)}{f(\eta)}}-\beta\right) \sin \chi \cos \phi_{\mathrm{pc}}\right\},
\end{aligned}
$$


where $\beta$ is defined right after eq. (19). Here we should reiterate that the formal usage of a general expression for $\Delta \rho=\rho_{\mathrm{GJ}}-\rho(=\Delta F / \alpha c S[\eta])$ to solve the Poisson's equation for arbitrarily large altitudes leads to the inconsistent and even erroneous result, mostly because of the physical reasons discussed in the end of previous Section. Namely, such a solution would imply the building up of the effective flux, $\Delta F$, exceeding the GJ flux $F_{\mathrm{GJ}}(\eta=1)$, fixed at the stellar surface, and charge reversal of the accelerating electric field at high altitudes. Also, we would like briefly comment on the space-charge-limited flow approximation at high altitudes in pulsars. Generally, the space-charge limitation occurs when the ejected charges reduce the accelerating potential drop boosting the initial particle energy. In pulsars, the flux of electrons ejected from the PC surface is limited by the value of GJ space charge at the bottom of the PC. In this case $F$ remains constant along the magnetic stream lines and is determined by $F_{\mathrm{GJ}}$ at $\eta=1$. Above the PC surface the space charge of ejected electrons reduces the "vacuum" potential drop by limiting it to the value determined by a small imbalance between the GJ charge density and actual charge density of electrons. This approximation is perfectly valid within a few stellar radii above the PC surface of most pulsars, when $|\Delta F(\eta)| \lesssim\left|F_{\mathrm{GJ}}(1)\right|$. However, at high enough altitudes, where $|\Delta F(\eta)|>\left|F_{\mathrm{GJ}}(1)\right|$ the situation is akin to the acceleration of test particles in a vacuum-like potential drop. Let us examine this in more detail for the SG by using the above expressions for $\Delta F$ and then formulate the derivation of an approximate but physically meaningful solution.

For low altitudes, $\eta \approx 1+z(z \ll 1)$, we can write

$$
\Delta F \approx 3 F_{0} z\left(\kappa \cos \chi+\frac{1}{4} H(1) \theta_{0} \sin \chi \cos \phi_{\mathrm{pc}}\right),
$$

and

$$
F_{\mathrm{GJ}} \approx F_{0}\left[(1-\kappa) \cos \chi+\frac{3}{2} H(1) \theta_{0} \sin \chi \cos \phi_{\mathrm{pc}}\right],
$$

so that $|\Delta F| \ll\left|F_{\mathrm{GJ}}\right|$, assuming that $F_{\mathrm{GJ}} \neq 0$. This means that at low altitudes the imbalance between $F$ and $F_{\mathrm{GJ}}$ (the flux of fictitious charges) that gives rise to the accelerating electric field in that region is much smaller than the local GJ flux, and therefore there will be no disruption of the steady-state regime of particle flow within the SG.

At large distances, $1 \ll \eta \ll \eta_{l c}$,

$$
|\Delta F| \approx\left|\Delta F_{*}(\eta, \xi)\right| \approx\left|F_{\mathrm{GJ}, 1}\right|,
$$

where $\left|F_{\mathrm{GJ}, 1}(\eta \gg 1)\right| \gg\left|F_{\mathrm{GJ}}(z=\eta-1 \ll 1)\right|$, and $F_{\mathrm{GJ}}(z \ll 1)$ is a function of $\xi$ only (i.e. is nearly a constant along the field lines). 
Thus, for low altitudes, up to approximately one-two stellar radii above the PC surface,

$$
F_{\mathrm{GJ}} \approx F_{\mathrm{GJ}, 0}
$$

and

$$
|\Delta F| \leq\left|F_{\mathrm{GJ}}\right| .
$$

Expressions (34), (35) imply that the SG boundaries can easily acquire the necessary surface charge by means of redistribution of space charges along the field lines in the vicinity of the SG boundaries, thus enabling the fulfillment of both the equipotentiality of SG boundaries and continuity of $\mathbf{E}_{\perp}$ across them. Note that, the component $\mathbf{E}_{\perp}$ and therefore the magnitude of induced surface charge is mostly determined by $F_{\mathrm{GJ}, 1}$ (or by $\rho_{\mathrm{GJ}, 1}$ ). Since in this region $\left|F_{G J, 1}\right| \leq\left|F_{G J, 0}\right|$, the required surface charge can be easily built up by establishing a weak current along the boundary field lines, without violating the GJ condition that in a steadystate regime the flux of charges from (to) the stellar surface should be limited by $F_{G J, 0}$. We must also note that the presence of a weak current along the SG boundary determined by flux $F_{\mathrm{GJ}, 1}$ is perfectly compatible with equipotentiality of the boundary. For example, this can be achieved by establishing a slightly non-homogeneous distribution of charges in a tiny skin layer along the boundary, at $\Phi=0$.

As we move up to higher altitudes along the SG boundaries where

$$
\left|F_{\mathrm{GJ}, 1}\right| \geq\left|F_{\mathrm{GJ}, 0}\right|,
$$

and where $\mathbf{E}_{\perp}^{\prime}$ is mostly determined by the term $\sim(\Omega \times \mathbf{r} / c) \times \mathbf{B}$, the situation changes dramatically. In this case the equipotentiality of the $\mathrm{SG}$ boundaries becomes fundamentally incompatible with a steady state regime. In other words, it is very unlikely that the SG boundaries can be steadily maintained in dynamic equilibrium in the presence of a strong $\mathbf{E}_{\perp}^{\prime}$ component. Rather, it is this region where the centrifugal-slingshot effect makes the electrons/positrons 'slip' from the magnetic field lines, and therefore effectively prevents charges from building up the otherwise required surplus surface charge on SG boundaries. Thus, in a steady-state situation the SG boundaries can be maintained as equipotential, if the approximate condition $\mathbf{E}_{\perp}^{\prime}=0$ (or its classical counterpart [15], as it will be referred to in the rest of the paper) is achieved throughout the SG outermost section. This means that in the outermost section of SG the value of $\Delta F(\eta, \xi)$ (see [30]) cannot grow (because of cross-field motion of charges at very high altitudes that effectively destroys the excessive GJ space charge in this region), and it is likely to nearly saturate at $\eta \approx \eta_{c}$ remaining constant along the stream lines. In this case the scalar potential $\Phi$ is described by the following Poisson's equation

$$
\nabla \cdot\left(\frac{1}{\alpha} \nabla \Phi\right)=-4 \pi \Delta \rho_{\mathrm{eff}}
$$

